\title{
Candidate cryovolcanic regions on Ganymede: a target priority for JUICE
}

\author{
Anezina Solomonidou ${ }^{1,2}$, Katrin Stephan ${ }^{3}$, Klara Kalousova ${ }^{4}$, and Krista Soderlund ${ }^{5}$ \\ ${ }^{1}$ California Institute of Technology (Caltech), Los Angeles, United States of America (anezina.solomonidou@jpl.nasa.gov) \\ ${ }^{2}$ LESIA-Observatoire de Paris, CNRS, UPMC Univ., Paris 06, Univ. Paris-Diderot, Meudon, France \\ ${ }^{3}$ Institute of Planetary Research, German Aerospace Center (DLR), Berlin, Germany \\ ${ }^{4}$ Charles University, Faculty of Mathematics and Physics, Department of Geophysics, Prague, Czech Republic \\ ${ }^{5}$ Institute for Geophysics, Jackson School of Geosciences, The University of Texas at Austin, Austin, USA
}

Jupiter's largest moon, Ganymede, is the main target of the upcoming ESA mission JUpiter ICy moons Explorer (JUICE), which is planned to launch in 2023. One of the top priorities of the JUICE mission is investigation of past and/or recent cryovolcanic and tectonic activity and the exchange processes with the subsurface and possibly with the ocean (Grasset et al., 2013). Following that objective, the science team has defined 'potential cryovolcanic regions' as a category of high interest for observation by JUICE (Stephan et al. 2021). Hence, for preparation of the scientific return of the mission, it is important to study in detail the regions that are considered to be good candidates for past or present activity.

Areas on Ganymede imaged by Voyager that showed the presence of dark terrain were speculated to represent a heavily cratered surface modified by cryovolcanism (e.g., Murchie et al., 1989); however, this was disputed based on the higher-resolution images of the Galileo mission (e.g., Pappalardo et al., 2004). Light material observed by the Voyager instruments was similarly suggested to represent dark terrain resurfaced by cryovolcanic flows (e.g., Parmentier et al., 1982). Later, Galileo high-resolution data showed the significant role of tectonism in the formation of these areas, while the role of cryovolcanism remained inconclusive due to the limited resolution of the available data and secondary processes that complicate their interpretation, such as fracturing, mass wasting, etc (e.g., Patterson et al., 2010). Currently, small, isolated depressions called 'paterae', are the best candidate regions for cryovolcanic activity on Ganymede and suggested to be potential caldera-like cryovolcanic source vents (Fig. 1;2)(e.g., Lucchita, 1980; Spaun et al. 2001). Collins et al. (2013) characterize the paterae as 'flat-floored depressions surrounded by inwardscalloped walls, breached on one side and typically associated with light subdued materials' while they interpret their nature as 'possible cryovolcanic source vents for extrusion of clean icy material to form light material units'. The small size of paterae (20 x $70 \mathrm{~km}$ at most) is consistent with a cryovolvanic origin that operates on a local scale.

The high-resolution JUICE camera, JANUS, in combination with other remote sensing instruments, is expected to resolve many of the mysteries concerning cryovolcanism on Ganymede and the origin of the moon's varied geologic features. The known paterae (Fig. 1) are located in smooth and bright terrains, where extensional tectonism and volcanism are speculated to have operated concurrently (Pappalardo et al. 2004). The 'potential cryovolcanic regions' identified by the JUICE team includes 
19 out of 30 paterae mapped by Collins et al. (2013) using Voyager and Galileo images. In this study, we provide a thorough view of all 19 paterae regions and a constructional comparison of their characteristics in order to constrain the morphology of the paterae and their surroundings, in preparation of the JUICE mission and its science return.

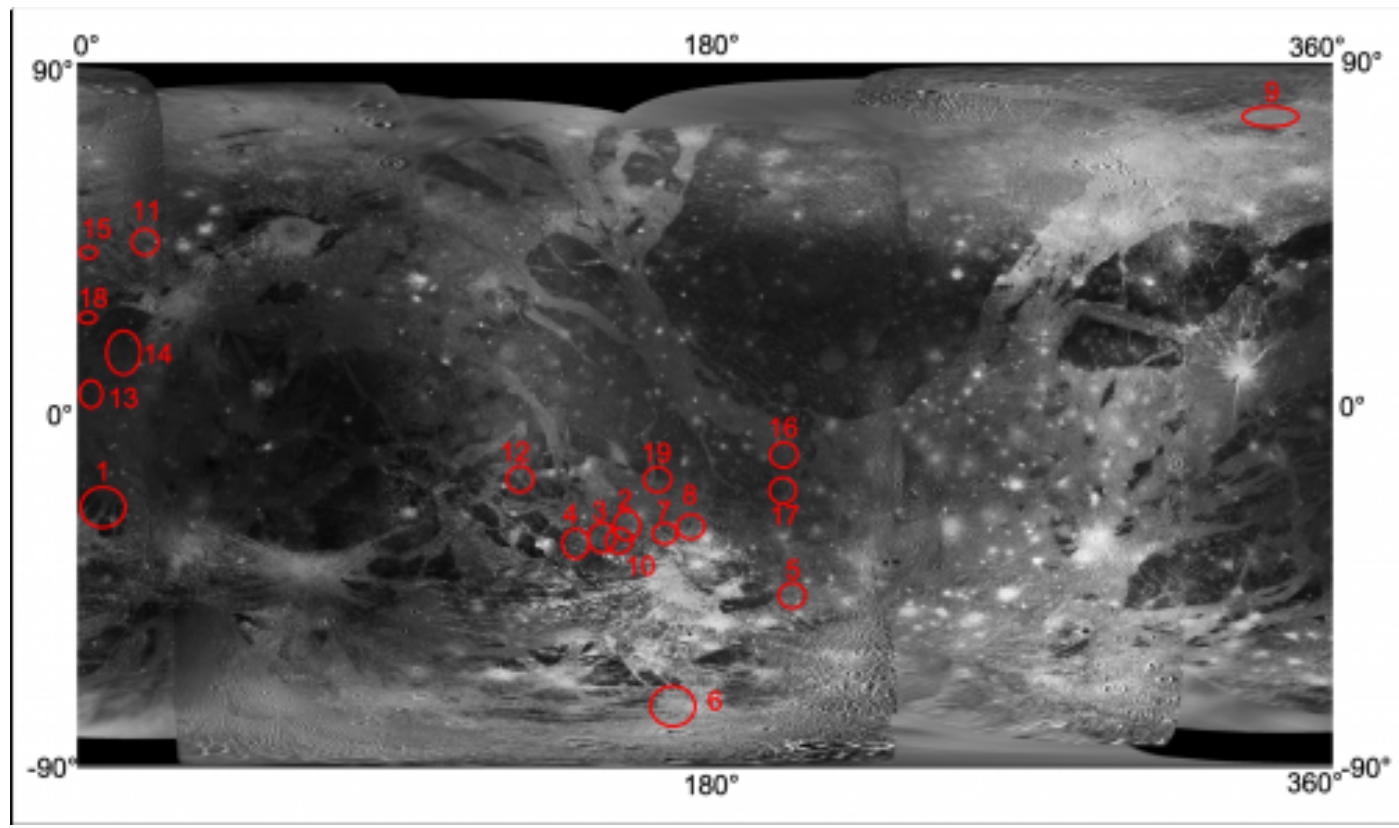

Figure 1. Locations (red circles and ellipsoids) of 19 paterae identified as 'potential cryovolcanic regions' that are of high interest for JUICE observations (Stephan et al. 2021). Basemap: Galileo Solid State Imaging (SSI)/Voyager Imaging Science Subsystem (ISS) mosaic.

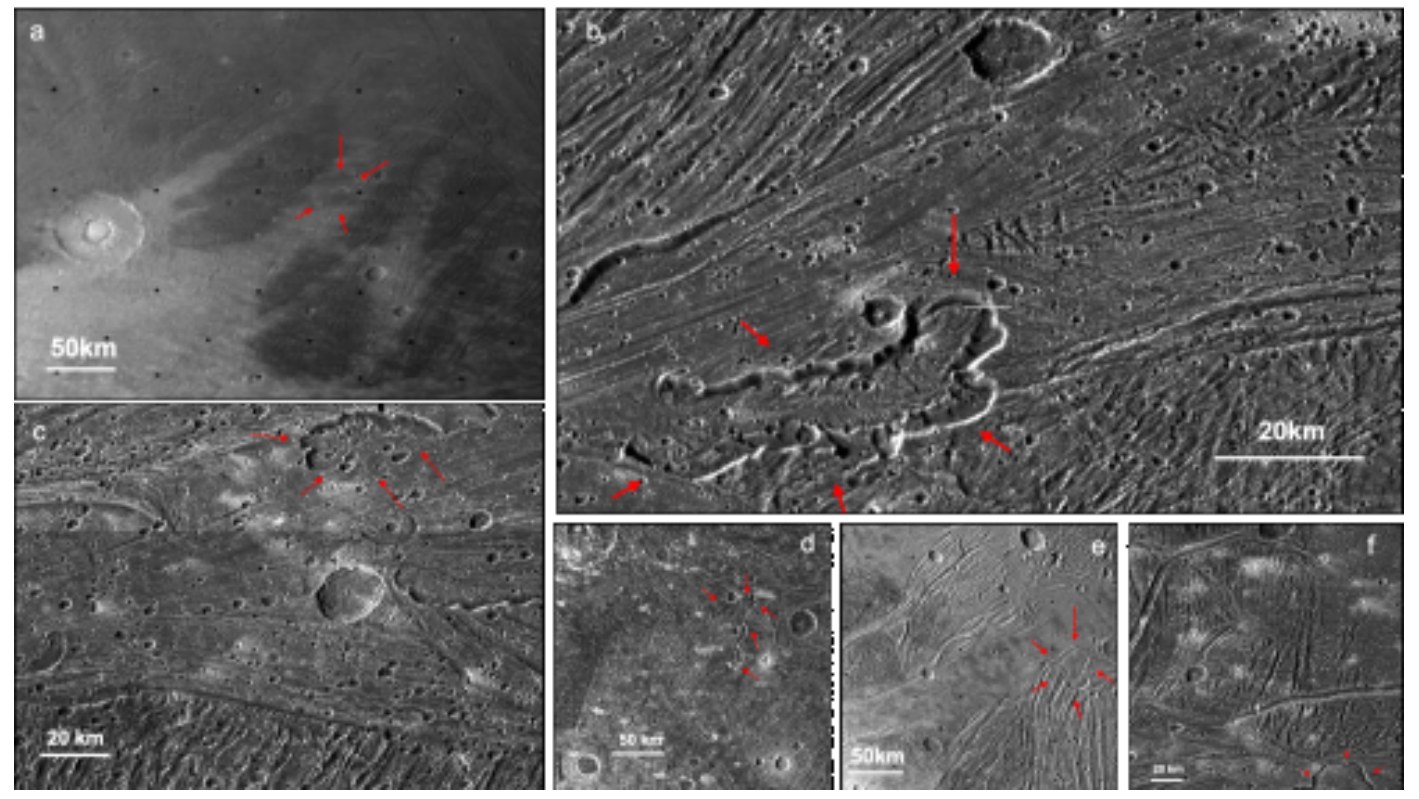

Figure 2. Major Ganymede Paterae as observed from the Voyager and Galileo instruments: a. Hammamat (1 in Fig. 1); b. Musa (4); c. Rum (2); d. Yaroun (5); e. Hamra (6); f. Natrun (2). Data from: Galileo Solid State Imaging (SSI)/Voyager Imaging Science Subsystem (ISS).

References: Grasset et al. (2013), PSS, 78, 1-21; Stephan et al. (2021), PSS in press; Murchie et al. (1989). Icarus, 81, 271-297; Pappalardo, R., et al. (2004), Cambridge, UK: Cambridge University 
Press; Parmentier, E., et al. (1982). Nature, 295, 290-293; Patterson, G., et al. (2010). Icarus, 207, 845-867; Lucchita, (1980). Icarus, 44, 481-501; Spaun et al. (2001), LPSC, 32; Collins, et al. (2013), USGS Scientific Investigations Map 3237, 4.

Acknowledgments: This work was conducted at the California Institute of Technology (Caltech) under contract with NASA. K.K. was supported by the Czech Science Foundation through project No. 19-10809S. (C2021 California Institute of Technology. Government sponsorship acknowledged. 